\title{
Triradiate deformity of the pelvis in Paget's disease of bone
}

\author{
N. K. Chakravorty \\ M.R.C.P.(E.), D.T.M. \& H. \\ Department of Geriatric Medicine, St Luke's Hospital, Huddersfield
}

\begin{abstract}
Summary
Possibly owing to the rather unusual manner of its presentation, there is scant description of triradiate deformity of the pelvis in textbooks. Three cases are now described to illustrate this feature of Paget's disease. Its approximate incidence is indicated.
\end{abstract}

\section{Introduction}

The usual features of Paget's disease of bone, large head, bowed legs, deafness due to involvement of the 8th cranial nerve, etc., are all well described in textbooks of medicine (Barry, 1969). Unlike a previous article (Chakravorty, 1978) which is of a more general nature, this article deals almost exclusively with the changes that occur in the pelvic region, i.e. triradiate deformity and protrusio acetabuli, features which have had very little description in the literature.

\section{Triradiate deformity and protrusio acetabuli}

The pelvis and femur are the most frequent sites of skeletal involvement in Paget's disease of bone (Dickson, Camp and Ghormley, 1945). The patient may occasionally complain of pain referable to the hip or the lower back. Most lesions are found incidentally during radiographic examination, which includes lower spines, pelvis, and upper femora.

Normally the pelvic inlet is somewhat heartshaped (Johnston, Davies and Davies, 1958), but softening of the pelvic bones may lead to pressure indentation of the pelvis opposite the hip joints. When the softening is less general and most marked in the acetabular region, the pressure of the femoral head deepens the acetabular cavity and causes a medial convex bulge of the wall of the pelvic cavity directly opposite, a condition called protrusio acetabuli. However, in an advanced case of Paget's disease, when both sides of the pelvis are affected, there may be a triradiate deformity of the pelvis due to pressure of the femoral heads on the softened acetabuli on both sides (Simon, 1973) (Fig. 1); in the case of protrusio acetabuli, the radiographic examination would show that only one side was affected.

It is worth noting that triradiate deformity of the pelvis may also result from other pathological con- ditions such as osteomalacia in adults, especially the elderly, and rickets in children.

The following cases are described to illustrate the triradiate deformity.

\section{Case 1}

This 85-year-old woman was seen by the author because of deafness, dementia and incontinence. She had a large head, and deformity of the legs. She scored 1/10 on the Mental Status Questionnaire Test. She was not able to walk. BP was $110 / 70$ $\mathrm{mmHg}$. Heart rate $84 / \mathrm{min}$ (regular). There was a systolic murmur in the aortic area. No evidence of congestive cardiac failure. No other neurological deficit apart from deafness and dementia. Examination of other systems was non-contributory.

Relevant investigations. $\mathrm{Hb}, 12.3 \mathrm{~g} / \mathrm{dl}$; WBC, $5 \cdot 2 \times$ $10^{9} / 1$; serum alkaline phosphatase, $79.5 \mathrm{KAu} . / \mathrm{dl}$ (normal, 3-13); serum calcium and inorganic phosphate normal; hydroxyproline/creatinine ratio, $0 \cdot 111$ (normal, <0.022). X-rays: chest, 'heart is enlarged, with the apex of the prominent left ventricle in the left axilla; lungs are clear; skull, 'widespread and severe changes of Paget's disease of the vault and the base producing a well marked basilar invagination'; pelvis and both hip joints, 'extensive Paget's disease involving the whole of the pelvis. The trabecular pattern is coarse and there is triradiate deformity of the pelvis. Gross osteo-arthritic changes are seen in both hip joints'.

\section{Case 2}

This 89-year-old man was admitted because of some difficulty in walking. There were no obvious features of Paget's disease, such as enlargement of the head or deformity of the legs, and he was not unduly deaf. BP, $150 / 80 \mathrm{mmHg}$; heart rate, $84 / \mathrm{min}$ (regular); no evidence of congestive cardiac failure; he scored 7/10 on the Mental Status Questionnaire Test, and he had no other significant neurological deficit. Examination of other systems was noncontributory.

Relevant investigations. $\mathrm{Hb}, 14 \mathrm{~g} / \mathrm{dl}$; WBC, $8.4 \times$ $10^{9} / 1$; serum alkaline phosphatase, $25.9 \mathrm{KAu} . / \mathrm{dl}$ 


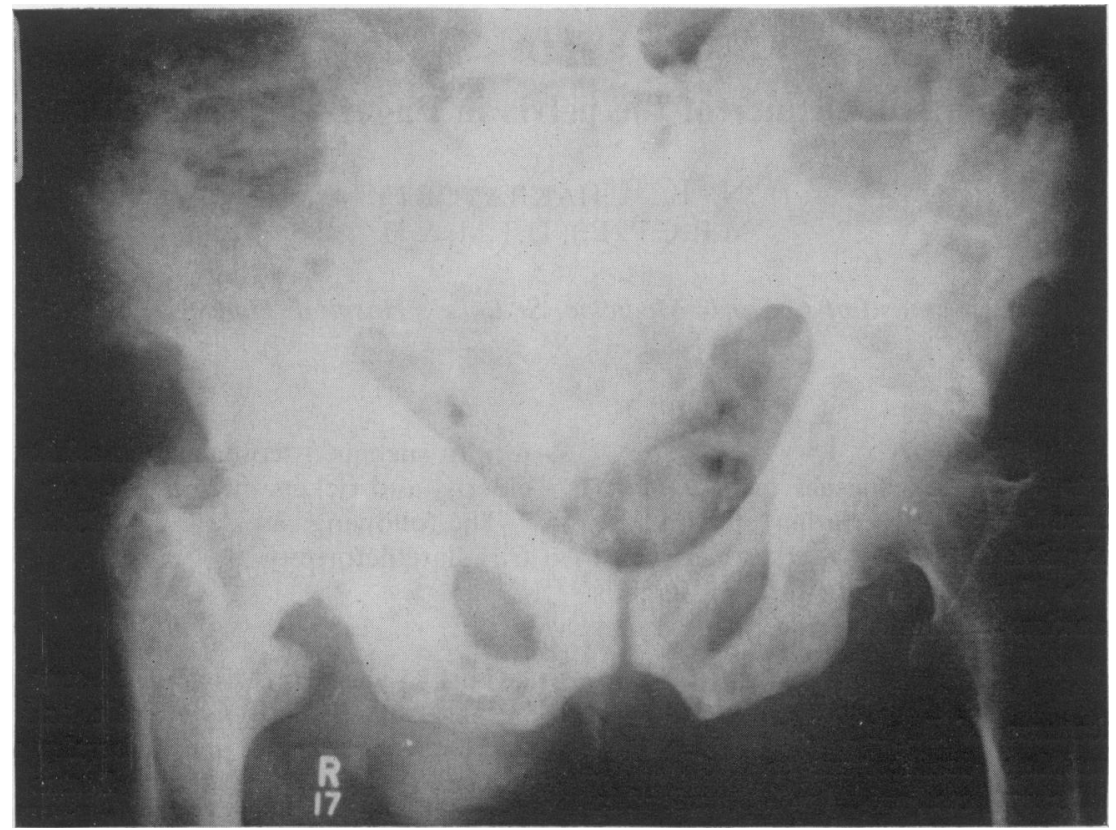

FIG. 1. X-ray of pelvis showing severe changes of Paget's disease involving both sides of the pelvis and right upper femur. There is also triradiate deformity of the pelvis.

(normal, 3-13); serum calcium and inorganic phosphate, normal; hydroxyproline/creatinine ratio, 0.044 (normal, < 0.022). X-rays, chest, 'the heart is enlarged with prominence of the left ventricle; lung fields clear'; L-S and pelvis, 'there is marked Paget's disease in the L1, L3 and L5 with compression of L3. Severe changes are present in both sides of the pelvis with triradiate deformity, and in the right femoral shaft' (Fig. 1).

\section{Case 3}

This 77-year-old man presented with deafness, large head and bowing of the left leg. BP 140/80 $\mathrm{mmHg}$. He had cardiomegaly with an aortic systolic murmur, but no evidence of congestive cardiac failure. No other neurological deficit apart from deafness. Examination of other systems was noncontributory.

Relevant investigations. Serum calcium and inorganic phosphate normal, but serum alkaline phosphatase was raised to $178 \mathrm{KAu}$./dl (normal, 3-13); urinary hydroxyproline $2.024 \mathrm{mmol} / 1$; hydroxyproline/creatinine ratio, 0.188 (normal, $<0.022$ ). $\mathrm{X}$-rays: chest, 'the heart is enlarged and of hypertensive contour and the aortic arch is unfolded. The lung fields clear'; bones, 'evidence of Paget's disease in the shoulder girdles, extensive Paget's disease of the skull, with a Tam O'Shanter skull, gross Paget's disease of the pelvis with triradiate deformity'.

\section{Case 4}

A 76-year-old man having closely similar clinica衰 features to case 3 .

\section{Discussion}

While biochemical data, such as a rise in serum alkaline phosphatase and urinary excretion of hydroxyproline, in the above cases were characteristic of Paget's disease of bone, only radiographic examination of the pelvis provided the conclusive evidence of triradiate deformity.

It will be seen that the patients were elderly, i.e. aged 76 and over, and that they represented Paget's disease in an advanced stage. It is possible that tri- 3 radiate deformity is more common in such cases. Deformity of the legs cannot be regarded as a reliable indication of associated triradiate deformity of the pelvis, since case 2 had no deformity of the legs. Out of a total of 534 patients admitted under the author $\sigma$ during 1978, there were 21 cases of Paget's disease, N 13 with pelvic involvement and, of these, 4 showed N definite radiological evidence of triradiate deformity. This suggests that the incidence of triradiate deformity may be approximately $0.75 \%$.

\section{Acknowledgments}

I wish to thank Dr R. R. Pritchard for his assistance in $\bar{O}$ preparing the manuscript. I also wish to thank Mr Morris, $\mathbb{D}$ 
Medical Photographer, Mrs Sheard, Medical Librarian, Mrs Hilton, Medical Secretary, and Sister Noble, for their assistance.

\section{References}

BARRY, H.C. (1969) Paget's Disease of Bone. Churchill Livingstone, Edinburgh.

Chakravorty, N.K. (1978) Some unusual features of Paget's disease of bone. Gerontology, 24, 459.
Dickson, D.D., CAMP, J.D. \& Ghormley, R.K. (1945) Osteitis deformans: Paget's disease of bone. Radiology, 44, 449.

Greenfield, G.B. (1975) Radiology of Bone Disease, 2nd edn, p. 81. J. B. Lippincott, Philadelphia.

Johnston, T.B., Davies, D.V. \& Davies, F. (1958) Gray's Anatomy, 32nd edn, p. 404. Longmans, London.

Simon, G. (1973) Principles of Bone X-Ray Diagnosis. 3rd edn, pp. 104 \& 179. Butterworth, London. 